Particularly interesting is the presence of both a stable, broadband frequency component and a variable narrowband component that is associated with longitudes of high reflectivity (see review paper for implications on surface characteristics).

In the fall of 1964 radar observations of Jupiter were attempted at a wavelength of $70 \mathrm{~cm}$. No significant echoes were observed.

The assistance of A. D. Sanchez and D. P. Campbell is specifically acknowledged.

Discussion following R. B. Dyce's paper

$J$. Warwick: Can these negative observations be expressed, in the absence of absorption, as an upper limit to the Jovian ionospheric electron density?

R. B. Dyce: Yes. A critical reflection model would set an upper limit of $2 \times 10^{9}$ electrons $-\mathrm{cm}^{-3}$.

J. Warwick: Are there plans to employ the future 40-Mc/s Arecibo radar system to such a search for a Jovian ionosphere?

G. H. Pettengill: The lower antenna gain and higher sky noise will cause the $40-\mathrm{Mc} / \mathrm{s}$ system to be about $20 \mathrm{~dB}$ less sensitive than the $430-\mathrm{Mc} / \mathrm{s}$ system.

R. Goldstein: No Jupiter echoes were detected by the JPL equipment in 1964 using an identical experimental configuration as in 1963.

(Paper 69D12-613)

\title{
Radio Evidence on the Structure and Composition of the Martian Surface
}

\author{
Carl Sagan \\ Harvard University \\ and \\ Smithsonian Astrophysical Observatory, Cambridge, Mass. \\ and \\ James B. Pollack \\ Smithsonian Astrophysical Observatory, Cambridge, Mass.
}

The radar observations of Mars near the 1963 and 1965 oppositions are discussed. In the 1963 observations of Goldstein and Gillmore, near planetocentric latitude $13^{\circ}$ north, a significant correlation is noted of radar reflectivity enhancements with the Martian dark areas, Cerberus Trivium Charontis, Nepenthes, and Syrtis Major. Limonite, $\mathrm{Fe}_{2} \mathrm{O}_{3} \cdot n \mathrm{H}_{2} \mathrm{O}$, is suspected to be a predominant constituent of the Martian bright areas on the basis of a variety of colorimetric, polarimetric, and infrared spectrometric observations. The values of the general radar reflectivity of the Martian bright areas are consistent with the identification of limonite on Mars only if the limonite is pulverized down to a depth of some meters. The deduced degree of pulverization of Martian limonite has been found to be achieved easily by pulverized limonite in the laboratory. The Martian dark areas are probably porous to a depth of meters, but their porosity is less than that of the bright areas. If limonite is a major constituent of the Martian bright areas, then passive microwave observations at wavelengths longward of $1 \mathrm{~cm}$ refer to a depth at which the diurnal thermal wave is damped to negligibly small amplitude. The mutual consistency of the infrared and microwave brightness temperatures of Mars is discussed.

(Paper 69D12-614) 